\title{
Memory effect and BMS-like symmetries for impulsive gravitational waves
}

\author{
Srijit Bhattacharjee $\odot^{*}$ and Shailesh Kumar ${ }^{\dagger}$ \\ Indian Institute of Information Technology (IIIT), Allahabad, \\ Devghat, Jhalwa, Uttar Pradesh-211015, India \\ Arpan Bhattacharyya \\ Yukawa Institute for Theoretical Physics (YITP), Kyoto University, \\ Kitashirakawa Oiwakecho, Sakyo-ku, Kyoto 606-8502, Japan
}

(Received 5 June 2019; revised manuscript received 30 August 2019; published 7 October 2019)

\begin{abstract}
Cataclysmic astrophysical phenomena can produce impulsive gravitational waves that can possibly be detected by the advanced versions of present-day detectors in the future. The gluing of two spacetimes across a null surface produces impulsive gravitational waves (in the phraseology of Penrose) having a Dirac Delta function type pulse profile along the surface. It is known that Bondi-van der Burg-Matzner-Sachs (BMS)-like symmetries appear as soldering freedom while we glue two spacetimes along a null surface. In this paper, we study the effect of such impulsive gravitational waves on test particles (detectors) or geodesics. We show explicitly some measurable effects that depend on BMS-transformation parameters on timelike and null geodesics. BMS-like symmetry parameters carried by the gravitational wave leave some "memory" on test geodesics upon passing through them.
\end{abstract}

DOI: 10.1103/PhysRevD.100.084010

\section{INTRODUCTION}

The "memory effect" for gravitational waves [1-6] has attracted considerable attention in recent times due to (i) its theoretical connection with asymptotic symmetries and soft theorems [7,8], and (ii) possibility of its detection in the advanced detectors like aLIGO [9] or in LISA [10-14]. The gravitational memory effect is described as permanent displacement (or change in velocity) of a system of freely falling particles (idealized detectors), initially at relative rest, upon passing of a burst of gravitational radiation. In asymptotically flat spacetimes, in the far region, this change can be related to the diffeomorphisms that preserve the asymptotic structure, i.e., with the action of the Bondi-van der Burg-MatznerSachs (BMS) group [15]. Further, it has been shown that this displacement or change in metric components due to the passage of gravitational radiation is just the Fourier transform of the Ward identity satisfied by the infrared sector (soft) of quantum gravity's $S$ matrix, corresponding to the BMS-like symmetries (like supertranslations).

\footnotetext{
*srijuster@gmail.com

shaileshkumar.1770@gmail.com

*bhattacharyya.arpan@yahoo.com
}

Published by the American Physical Society under the terms of the Creative Commons Attribution 4.0 International license. Further distribution of this work must maintain attribution to the author(s) and the published article's title, journal citation, and DOI. Funded by SCOAP ${ }^{3}$.
These findings and Hawking-Perry-Strominger's [16,17] proposal-that black holes may possess an infinite number of soft hairs corresponding to the spontaneously broken supertranslation symmetry - has generated a lot of activities towards finding the BMS-like symmetries near the horizon of black holes. However, this has already been accomplished in various ways. The BMS group and its extended version (including superrotation) [18] have been recovered by analyzing the diffeomorphisms that preserve the near horizon asymptotic structure of black holes $[19,20]$. Recently, it has been shown that the BMS symmetries can be recovered at any null hypersurface situated at some finite location of any spacetime [21]. In the context of soldering of two spacetimes across a null hypersurface (like black hole horizons), BMS-like symmetries have been recovered in $[22,23]$. Now a natural question arises: Is it possible to detect some memory effect near the horizon of a black hole or at a null surface placed at some finite location of the spacetime just like what was found in the far region? In terms of the emergence of extended symmetries near the horizon of a black hole, BMS memory has been indicated in [24]. The purpose of this paper is to study the gravitational memory effect at a finite location of spacetime and obtain some detectable features on test particles or geodesics.

Memory effect for plane gravitational waves has been extensively studied in recent times $[25,26]$. The search for memory effect has also been extended to impulsive plane 
gravitational waves [27]. ${ }^{1}$ Memory effect for plane-fronted impulsive waves on null geodesic congruence (massless test particles) has been found in [29], where BMS supertranslation-type memory has been obtained. We extend these studies for timelike geodesics (test particles or detectors). We show both supertranslation and superrotation-like memory effects, when a pulse of the gravitational wave crosses these test particles. We also show that there are jumps in expansion and shear for a null congruence encountering impulsive gravitational waves (IGWs).

An IGW is an artifact of Penrose's "cut and paste" method to glue two spacetimes [30]. Mathematically speaking, these are spacetimes having a Riemann tensor containing a singular part proportional to the Dirac delta function whose support is a null or lightlike hypersurface. This kind of signal can be represented unambiguously as a pure gravitational wave part and a shell or matter part. Such impulsive signals are produced during violent astrophysical phenomena like supernova explosion or a coalescence of black holes. Naturally studying the effect of such IGW on detectors (placed nearby the source) would be important from an astrophysical perspective also. In this paper, we consider null hypersurfaces that represent the history of the IGW, generated by gluing two spacetimes across the null surface and study the effect of these bursts of radiation on geodesics crossing it. As shown in [22] and [23], the gluing is not unique and one can construct infinitely many thin null shells related to each other via BMS-like transformations (on either side of the surface). For this reason, the geodesics crossing the IGW suffer a memory effect induced by BMS symmetries.

In Sec. II, we review singular null shells in general relativity and show how BMS-like soldering freedom arises when one tries to glue two spacetimes across a null hypersurface. In Sec. III, we study the memory effect for IGW on timelike geodesics (detectors). We show how the relative position of test particles changes due to the passage of such a pulse signal. Next, we study the effect of IGW on null congruences. Here we calculate the jump in optical properties of geodesic congruence, namely, shear and expansion parameters due to the passage of IGW indicating memory effect. Finally, we conclude with the discussion of the outcomes and future objectives.

\section{IGW AND EMERGENCE OF BMS INVARIANCE ON NULL SHELLS}

IGWs are generated when one tries to glue two spacetimes across a null hypersurface. To see this, let us consider two manifolds $\mathcal{M}_{+}$and $\mathcal{M}_{-}$, with corresponding intrinsic metrics $g_{\mu \nu}^{+}\left(x_{+}^{\mu}\right)$ and $g_{\mu \nu}^{-}\left(x_{-}^{\mu}\right)$. Throughout this paper, we will work in four dimensions. We use greek alphabets for

\footnotetext{
${ }^{1}$ See [28] for clarifications on the calculation method adopted in [27].
}

spacetime indices. Latin lowercase and uppercase letters are used for hypersurface and codimension-1 surface quantities respectively. Suppose a common coordinate system $x^{\mu}$ that has overlaps with $x_{ \pm}^{\mu}$ is installed across the common null boundary $\Sigma$ of $\mathcal{M}_{ \pm}$. We set $\left.x^{\mu}\right|_{\Sigma}=\zeta^{a}$. Let $e_{a}^{\mu}=\partial x^{\mu} / \partial \zeta^{a}$ be tangent vectors to $\Sigma$, and $n$ is a normal vector to the hypersurface defined as $n^{\alpha}=g^{\alpha \beta} \partial_{\beta} \Phi(x)$, where the equation of $\Sigma$ is given by $\Phi=0$. The sign of this normal is taken so that it points to the future of $\Sigma$. We also have to define an auxiliary null vector $N^{ \pm}$to complete the basis such that $[31,32]$

$$
\left.N \cdot N\right|_{ \pm}=0, \quad n \cdot N=-1, \quad N_{\mu} \cdot e_{A}^{\mu}=0 .
$$

The junction condition in terms of common coordinates states

$$
\left[g_{a b}\right]=g_{a b}^{+}-g_{a b}^{-}=0
$$

together with

$$
\left[e_{a}^{\mu}\right]=\left[n^{\mu}\right]=\left[N^{\mu}\right]=0 .
$$

In terms of common coordinates, the metric reads

$$
g_{\mu \nu}=g_{\mu \nu}^{+} \mathcal{H}(\Phi)+g_{\mu \nu}^{-} \mathcal{H}(-\Phi),
$$

where $\mathcal{H}(\Phi)$ is the Heaviside step function. The Riemann tensor takes the following form upon using the junction condition (2.2):

$R_{\beta \gamma \delta}^{\alpha}=R_{\beta \gamma \delta}^{+\alpha} \mathcal{H}(\Phi)+R_{\beta \gamma \delta}^{-\alpha} \mathcal{H}(-\Phi)+\delta(\Phi) Q_{\beta \gamma \delta}^{\alpha}$,

where $Q^{\alpha}{ }_{\beta \gamma \delta}=-\left(\left[\Gamma_{\beta \delta}^{\alpha} n_{\gamma}-\left[\Gamma_{\beta \gamma}^{\alpha}\right] n_{\delta}\right)\right.$. Clearly, any nonvanishing component of $Q^{\alpha}{ }_{\beta \gamma \delta}$ indicates the existence of an impulsive gravitational wave supported on the null hypersurface $\Sigma$. Now, one can show that the glued spacetime satisfies Einstein's equation with the following stress tensor [31-34]:

$$
T_{\alpha \beta}=T_{\alpha \beta}^{+} \mathcal{H}(\Phi)+T_{\alpha \beta}^{-} \mathcal{H}(\Phi)+S_{\alpha \beta} \delta(\Phi)
$$

where $S_{a b}=S_{\alpha \beta} e_{a}^{\alpha} e_{b}^{\beta}$ is denoted as the stress tensor of the null hypersurface. The stress tensor $S^{\alpha \beta}$ can be expressed as [31-34],

$$
S^{\alpha \beta}=\mu n^{\alpha} n^{\beta}+j^{A}\left(n^{\alpha} e_{A}^{\beta}+e_{A}^{\alpha} n^{\beta}\right)+p \sigma^{A B} e_{A}^{\alpha} e_{B}^{\beta},
$$

where $\sigma_{A B}$ is the metric of the spatial slice of the surface of the null shell and $A, B$ denote the spatial indices of the null surface. $\mu, J^{A}$, and $p$ are the surface energy density, surface current (anisotropic stress), and pressure of the shell, respectively. Note that the null hypersurface has now 
become a shell with some null matter supported on it. We identify the intrinsic quantities of the shell as follows:

$\mu=-\frac{1}{8 \pi} \sigma^{A B}\left[\mathcal{K}_{A B}\right], \quad J^{A}=\frac{1}{8 \pi} \sigma^{A B}\left[\mathcal{K}_{V B}\right], \quad p=-\frac{1}{8 \pi}\left[\mathcal{K}_{V V}\right]$,

where

$$
\gamma_{a b}=N^{\alpha}\left[\partial_{\alpha} g_{a b}\right]=2\left[\mathcal{K}_{a b}\right]
$$

and $\mathcal{K}_{a b}=e_{a}^{\alpha} e_{b}^{\beta} \nabla_{\alpha} N_{\beta}$ is the oblique extrinsic curvature. The jump in the partial derivative of $g_{\mu \nu}$ is proportional to $\gamma_{\mu \nu}$, which contains all the necessary information of the properties of the shell:

$$
\left[\partial_{\lambda} g_{\mu \nu}\right]=\gamma_{\mu \nu} n_{\lambda}
$$

Next, we briefly review how BMS symmetries emerge while one tries to solder two spacetimes across a null hypersurface. As depicted in [22,23], if one tries to find possible coordinate transformations that preserve the induced metric on a null hypersurface across which two spacetimes are glued, BMS-like symmetry transformations emerge. This amounts to solving the Killing equation for $g_{a b}$, the induced metric on $\Sigma$ [22].

$$
\mathcal{L}_{Z} g_{a b}=0 .
$$

(i) Supertranslation-like freedom on the Killing horizon

We work in Kruskal coordinates $\left(U, V, x^{A}\right)$. Coordinates for the horizon $\Sigma$ are $\zeta^{a}=\left\{V, x^{A}\right\}$, where $V$ is the parameter along the hypersurface generating null congruences. In this coordinate the normal to the surface becomes $n^{\alpha}=\left(\partial_{V}\right)^{\alpha}$. Let us first consider the Schwarzschild horizon,

$d s^{2}=-2 G(r) d U d V+r^{2}(U, V)\left(d \theta^{2}+\sin ^{2}(\theta) d \phi^{2}\right)$,

where $G(r)=\frac{16 m^{3}}{r} e^{-r / 2 m}, \quad U V=-\left(\frac{r}{2 m}-1\right) e^{r / 2 m}$. The null shell is located at the Killing horizon $U=0$. The Killing equation (2.11) becomes

$Z^{V} \partial_{V} g_{A B}+Z^{C} \partial_{C} g_{A B}+\partial_{A} Z^{C} g_{B C}+\partial_{B} Z^{C} g_{A C}=0$,

leading $Z^{V}=F(V, \theta, \phi)$. Furthermore, if one also demands $\mathcal{L}_{Z} n^{a}=0$, then it gives

$$
\partial_{V} Z^{V}=0 .
$$

This will further restrict the form of $Z^{V}$ as $Z^{V}=T(\theta, \phi)$. Clearly, $Z$ generates the following symmetry:

$$
V \rightarrow V+T(\theta, \phi),
$$

and this is strikingly similar to the supertranslation found at the null infinity of asymptotically flat spacetimes [15]. This construction has been extended for the rotating black hole spacetimes in [23].

(ii) Superrotation-like soldering freedom

If the nondegenerate subspace of a null surface is parametrized by some null parameters (e.g., null coordinates $U$ or $V$ ) then (2.13) offers a new kind of solution. This has been shown in [23]. This situation gives rise to superrotations or the conformal rescaling of the base space of the null hypersurface. To see this, consider the following metric [23]:

$d s^{2}=\frac{2\left(\frac{V}{1+\eta \zeta \bar{\zeta}}\right)^{2} d \zeta d \bar{\zeta}+2 d U d V-2 \eta d U^{2}}{\left(1+\frac{\Lambda U}{6}(V-\eta U)\right)^{2}}$.

This metric can represent de Sitter space when $\Lambda>0, \eta=1$, anti-de Sitter space when $\Lambda<0$, $\eta=-1$, and Minkowski space when $\Lambda=0, \eta=0$. The null surface is at $U=0$. For this case, the following soldering freedoms are obtained [23]:

$$
\begin{array}{r}
V \rightarrow V\left(1-\frac{\epsilon \tilde{\Omega}(\zeta, \bar{\zeta})}{2}\right), \\
\zeta \rightarrow \zeta+\epsilon h(\zeta), \quad \bar{\zeta} \rightarrow \bar{\zeta}+\epsilon \bar{h}(\bar{\zeta}),
\end{array}
$$

where

$$
\begin{aligned}
& \tilde{\Omega}(\zeta, \bar{\zeta}) \\
& =\frac{(1+\eta \zeta \bar{\zeta})\left(h^{\prime}(\zeta)+\bar{h}^{\prime}(\bar{\zeta})\right)-2 \eta \bar{\zeta} h(\zeta)-2 \eta \zeta \bar{h}(\bar{\zeta})}{1+\eta \zeta \bar{\zeta}} .
\end{aligned}
$$

$h(\zeta)$ and $\bar{h}(\bar{\zeta})$ are holomorphic and antiholomorphic functions and $\epsilon$ is a small parameter. This kind of local conformal transformations are usually referred to as superrotation in the context of asymptotic symmetries. The emergence of such symmetries in the context of Penrose's impulsive wave spacetime (introducing snapping cosmic string) has also been discussed in [35].

\section{(iii) Null surface near the Killing horizon}

Superrotations can also be recovered when a null surface is situated just a little away from the 
horizon of a black hole [23]. Using similar types of coordinates as in Eq. (2.16) the two-dimensional spatial slice of the Schwarzschild black hole becomes $r^{2} \frac{d \zeta d \bar{\zeta}}{(1+\zeta \bar{\zeta})^{2}}$, with $r^{2}=4 m^{2}+\left(-\frac{8 m^{2}}{e}\right) U V+$ $\mathcal{O}(U V)^{2}+\cdots$. A null shell located at $U=\epsilon$ allows the following soldering transformations:

$$
\begin{aligned}
V & \rightarrow V(1-\tilde{\Omega}(\zeta, \bar{\zeta}))-\frac{a \tilde{\Omega}(\zeta, \bar{\zeta})}{b \epsilon}+\mathcal{O}(\epsilon), \\
\zeta & \rightarrow \zeta+h(\zeta), \quad \bar{\zeta} \rightarrow \bar{\zeta}+\bar{h}(\zeta) .
\end{aligned}
$$

Hence, when we glue a supertranslated (or superrotated) metric with a seed metric (e.g., Schwarzschild), these BMS-like symmetries emerge in the shell's intrinsic quantities as well as in the IGW part (see Appendix A).

\section{MEMORY EFFECT: TIMELIKE CONGRUENCE}

In this section, we study the interaction between IGWs and test particles following timelike geodesics. We must find some observable effects on the relative motion of neighboring test particles. To see this, we consider two test particles whose worldlines are passing through a null hypersurface supporting IGWs. The effect of IGWs on the separation vector of these nearby geodesics can be captured by the use of the geodesic deviation equation and junction conditions. The theory of impulsive gravitational waves is a well-studied area of research [33,36-38]. Usually, two different approaches are adopted. In [27,36-38] etc., a distributional metric is used and no null shell is introduced. Here, we use a local coordinate system in which the metric tensor is continuous but its first derivative has discontinuity across the null surface. As a result, we get a thin null shell along with the wave signal. It should be noted, the treatment presented here is based on the fact that a consistent $C^{0}$ matching of the metrics can be done maintaining the $C^{1}$ regularity of the geodesics across the null shells [39]. Rigorous mathematical treatment of IGW spacetimes addressing the existence, uniqueness, and generic properties of geodesics can be found in [40-43]. Here we closely follow the construction given in $[33,34]$ and review the necessary parts.

Let us consider a congruence having $T^{\mu}$ be the tangent vector, where $T^{\mu}$ is a unit timelike vector field

$$
g_{\mu \nu} T^{\mu} T^{\nu}=-1 .
$$

The integral curves of $T^{\mu}$ happen to be timelike geodesics. Therefore,

$$
\dot{T}^{\mu}=T^{\nu} \nabla_{\nu} T^{\mu}=0 .
$$

The dot denotes the covariant derivative of a tensor field in the $T^{\mu}$ direction. This congruence is passing through the null shell located at $\Sigma$. Now we introduce a separation vector $X^{\mu}$ satisfying $g_{\mu \nu} T^{\mu} X^{\nu}=0$ such that,

$$
\dot{X}^{\mu}=X^{\nu} \nabla_{\nu} T^{\mu} .
$$

This vector $X^{\mu}$ satisfies the geodesic deviation equation,

$$
\ddot{X}^{\mu}=-R_{\lambda \sigma \rho}^{\mu} T^{\lambda} X^{\sigma} T^{\rho} \text {. }
$$

$R_{\lambda \sigma \rho}^{\mu}$ is Riemann tensor of full spacetime $\mathcal{M}$. To be consistent with the jump in the transverse derivative of the metric (2.9), which produces a Delta function term in a Riemann tensor, we can assume the jumps in partial derivatives of $T^{\mu}$ and $X^{\mu}$ across $\Sigma$, and are given by

$$
\left[T_{, \lambda}^{\mu}\right]=P^{\mu} n_{\lambda} ; \quad\left[X_{, \lambda}^{\mu}\right]=W^{\mu} n_{\lambda},
$$

${ }^{2}$ for some vectors $\mathcal{P}$ and $W$ defined on $\Sigma$. The vectors $P, W$ are not necessarily tangential to $\Sigma$. Let $\left\{E_{a}\right\}$ be a triad of vector fields defined along the timelike geodesics tangent to $T^{\mu}$ by parallel transporting $\left\{e_{a}\right\}$ along these geodesics. Therefore,

$$
\dot{E}_{a}^{\mu}=0
$$

where $\left.E_{a}\right|_{\Sigma}=e_{a}$. Consequently, the jump in the partial derivatives of $E_{a}^{\mu}$ should take the following form for some $F_{a}^{\mu}$ defined on $\Sigma$ :

$$
\left[E_{a, \lambda}^{\mu}\right]=F_{a}^{\mu} n_{\lambda} .
$$

Let $X_{(0)}^{\mu}$ be the vector $X^{\mu}$ evaluated on $\Sigma$, i.e., the value of separation vector $X^{\mu}$ at the intersection point of the null hypersurface with the timelike congruence. Similarly, let $T_{(0)}^{\mu}$ denote the tangent vector $T^{\mu}$ evaluated on $\Sigma$. We can write $X_{(0)}^{\mu}$ in terms of components along the orthogonal and the tangential vectors to the hypersurface in the following way:

$$
X_{(0)}^{\mu}=X_{(0)} T_{(0)}^{\mu}+X_{(0)}^{a} e_{a}^{\mu},
$$

for some function $X_{(0)}$, and $X_{(0)}^{a}$ is the $X^{a}$ evaluated at $\Sigma$. Now if $\Sigma$ is located at $U=0$ (this happens typically in Kruskal-like coordinates), then using (3.5) and expanding around $U=0$ (keeping only the linear term), we get

$$
X^{\mu}=X^{-\mu}+U \mathcal{H}(U) W^{\mu},
$$

with $X^{-\mu}$, in general, having dependence on $U$ (in the side) such that when $U=0, X^{-\mu}=X_{0}^{\mu}$. It is convenient to calculate $X^{\mu}$ in the basis $\left\{E_{a}^{\mu}, T^{\mu}\right\}$. Its nonvanishing components,

$$
X_{a}=g_{\mu \nu} X^{\mu} E_{a}^{\nu}
$$

\footnotetext{
2،," means derivative.
} 
Using (3.8) and (3.9), we calculate the above expression for small $U>0$ (in + side),

$$
X_{a}=\left(\tilde{g}_{a b}+\frac{1}{2} U \gamma_{a b}\right) X_{(0)}^{b}+U V_{(0) a}^{-}
$$

where $V_{(0) a}^{-}=\left.\frac{d X_{a}^{-}}{d u}\right|_{U=0}$ and $\tilde{g}_{a b}$ is given by [using the definition of $\gamma_{a b}$ in (2.9)]

$$
\tilde{g}_{a b}=g_{a b}+\left(T_{(0) \mu} e_{a}^{\mu}\right)\left(T_{(0) \nu} e_{b}^{\nu}\right) .
$$

The last term in Eq. (3.11) denotes the relative displacement of test particles for small $U$ when no signals were present. As the $\tilde{g}_{a b}$ is nondegenerate we can invert it,

$$
X_{a}=\tilde{g}_{a b} X^{b}, \quad X^{a}=\tilde{g}^{a b} X_{b}
$$

To see the effect of the wave and shell part of the signal separately we decompose $\gamma_{a b}$ into a transverse-traceless part, and rest as

$$
\gamma_{a b}=\hat{\gamma}_{a b}+\bar{\gamma}_{a b}
$$

with

$$
\begin{aligned}
\bar{\gamma}_{a b}= & 16 \pi\left(g_{a c} S^{c d} N_{d} N_{b}+g_{b c} S^{c d} N_{d} N_{a}-\frac{1}{2} g_{c d} S^{c d} N_{a} N_{b}\right. \\
& \left.-\frac{1}{2} g_{a b} S^{c d} N_{c} N_{d}\right)
\end{aligned}
$$

where $S^{a b}$ is defined in (2.7) and we have also used the definitions (2.8). Here

$\hat{\gamma}_{a b}=\gamma_{a b}-\frac{1}{2} g_{*}^{c d} \gamma_{c d} g_{a b}-2 n^{d} \gamma_{d(a} N_{b)}+\gamma^{\dagger} N_{a} N_{b}$,

and $g_{*}^{a b}$ is the pseudoinverse of $g_{a b}$ where entries at the $V$ direction are zero defined as [31]

$$
g_{*}^{a b} g_{b c}=\delta_{c}^{a}-n^{a} N_{c}, \quad g_{*}^{c d} \gamma_{c d}=g^{A B} \gamma_{A B}
$$

Also, $\gamma^{\dagger}=\gamma_{a b} n^{a} n^{b}$ and $\gamma_{a b}$ is defined via (2.9). $\hat{\gamma}_{a b}$ encodes the pure gravitational wave degrees of freedom as $\hat{\gamma}_{a b} n^{b}=0=g_{*}^{a b} \hat{\gamma}_{a b}$. On the other hand, $\bar{\gamma}_{a b}$ encodes the degrees of freedom corresponding to the null matter of the shell. If $\bar{\gamma}_{a b}$ vanishes then we will have a pure impulsive gravitational wave. Otherwise, the IGW will be accompanied by some null matter.

Next, from (3.15) and using (2.8) for Kruskal-type coordinates we get

$$
\bar{\gamma}_{V B}=\bar{\gamma}_{B V}=16 \pi g_{B C} S^{V C}, \quad \bar{\gamma}_{A B}=-8 \pi S^{V V} g_{A B} .
$$

Using (3.13), (3.12), (3.15), and (3.16) in (3.11) we get

$$
\begin{aligned}
& X_{V}=X_{(0) V}+\frac{1}{2} U \bar{\gamma}_{V B} X_{(0)}^{B}+U V_{(0) V}^{-}, \\
& X_{A}=g_{A B} X_{(0)}^{B}+\frac{1}{2} U \gamma_{A B} X_{(0)}^{B}+U V_{(0) A}^{-} .
\end{aligned}
$$

We assume before the arrival of the signal, the test particles reside at the spacelike two-dimensional surface (signal front). Therefore, we must have

$$
X_{(0) V}=V_{(0) V}^{-}=0,
$$

leading to [using (3.18)]

$$
\begin{aligned}
& X_{V}=\frac{1}{2} U \bar{\gamma}_{V B} X_{(0)}^{B}=8 \pi U g_{B C} S^{V C} X_{(0)}^{B}, \\
& X_{A}=\left(1-4 \pi U S^{V V}\right)\left(g_{A B}+\frac{U}{2} \hat{\gamma}_{A B}\right) X_{(0)}^{B}+U V_{(0) A}^{-} .
\end{aligned}
$$

Note that, if the surface current $S^{V C} \neq 0$, then $X_{V} \neq 0$, which indicates that test particles will be displaced off the two-dimensional surface. Since $\hat{\gamma}$ does not affect $X^{V}$, this component of the deviation vector can only have a nonvanishing change if the IGW becomes the history of a nullshell containing current. Note that, for the plane-fronted wave we can have a shell without any matter, in which this part will be zero [33]. However, for spherical shells (such as the horizon shell in Schwarzschild spacetime), we cannot have a pure gravitational wave without matter [22,23]. For shells having $S^{V C}=0$, we only have the spatial parts of the deviation vector nonvanishing,

$$
X_{A}=\left(1-4 \pi U S^{V V}\right)\left(g_{A B}+\frac{U}{2} \hat{\gamma}_{A B}\right) X_{(0)}^{B} .
$$

The terms involving $\hat{\gamma}_{A B}$ in (3.22) describe the usual distortion effect of the wave part of the signal on the test particles in the signal front. However, there is an overall diminution factor in the first bracket of the above expression due to the presence of the null shell. We now examine the expression of deviation vector components for different cases.

\section{A. Memory effect and supertranslations at the black hole horizon}

We first consider the horizon shell of the Schwarzschild metric (2.12). For this case, following [22] we have

$\hat{\gamma}_{\theta \phi}=2 \frac{\nabla_{\theta}^{(2)} \partial_{\phi} F}{F_{V}}, \quad \hat{\gamma}_{\theta \theta}=\frac{1}{2}\left(\gamma_{\theta \theta}-\frac{1}{\sin ^{2} \theta} \gamma_{\phi \phi}\right)$.

If we focus on the case (2.15) for which we get supertranslation from the horizon soldering freedom, we get a shell having zero pressure and zero surface current, i.e., $S^{V A}=0=p$ [22]. Then we get 


$$
\begin{aligned}
& \hat{\gamma}_{\theta \phi}=2 \nabla_{\theta}^{(2)} \partial_{\phi} T(\theta, \phi), \\
& \hat{\gamma}_{\theta \theta}=2\left(\nabla_{\theta}^{(2)} \partial_{\theta} T(\theta, \phi)-\frac{1}{\sin ^{2} \theta} \nabla_{\phi}^{(2)} \partial_{\phi} T(\theta, \phi)\right) .
\end{aligned}
$$

Also the energy density becomes

$$
S^{V V}=-\frac{1}{32 m^{2} \pi}\left(\nabla^{2} T(\theta, \phi)-T(\theta, \phi)\right) .
$$

This shell allows impulsive gravitational waves along with some matter and the neighboring test particles will encode this into the $X^{A}$ components of the deviation vector (3.21) ( $X_{V}$ is zero):

$$
\begin{aligned}
X_{\theta}= & \left(1+\frac{U}{8 m^{2}}\left(\nabla^{2} T(\theta, \phi)-T(\theta, \phi)\right)\right) \\
& \times\left[\left(4 m^{2}+U\left(\nabla_{\theta}^{(2)} \partial_{\theta} T(\theta, \phi)\right.\right.\right. \\
& \left.\left.\left.-\frac{1}{\sin ^{2} \theta} \nabla_{\phi}^{(2)} \partial_{\phi} T(\theta, \phi)\right)\right) X_{(0)}^{\theta}+U \nabla_{\theta}^{(2)} \partial_{\phi} T(\theta, \phi) X_{(0)}^{\phi}\right]
\end{aligned}
$$

and similarly for the $X^{\phi}$ component.

Clearly, there is a distortion in the relative position of the particles after encountering the impulsive gravitational wave and the distortions are determined by the supertranslation parameter $T(\theta, \phi)$. The displacement is confined to the 2-surface and for physical matter [see (A6) of Appendix A] having a positive energy density, there will be a diminishing effect on the test particles. Hence, this is a reminiscence of the BMS memory effect (velocity) at future null infinity. We may integrate this expression with respect to the parameter of the geodesics to get a shift in the spatial direction and obtain the displacement memory effect. For a slowly rotating spacetime, we can get a similar kind of memory effect following [23].

\section{B. Memory effect and superrotation-like transformations}

To see the effect of superrotation on test particles, we first focus on "spherical" impulsive wave signal introduced by Penrose. The analysis for a hyperboloidal wave can be done similarly. Extending the transformation (2.17) off the null shell, we can compute the $\hat{\gamma}_{A B}$ and the stress tensor (2.7). The details of the computation are given in Appendix A. We simply quote the important results below.

$$
\hat{\gamma}_{\zeta \zeta}=\epsilon V h^{\prime \prime \prime}(\zeta), \quad \hat{\gamma}_{\bar{\zeta} \bar{\zeta}}=\epsilon V \bar{h}^{\prime \prime \prime}(\bar{\zeta})
$$

Also we get

$$
S^{V V}=0, \quad S^{V \zeta}=j^{\zeta}=\frac{\epsilon \bar{h}^{\prime \prime}(\bar{\zeta})}{16 \pi V^{2}}, \quad S^{V \bar{\zeta}}=j^{\bar{\zeta}}=\frac{\epsilon h^{\prime \prime}(\zeta)}{16 \pi V^{2}}
$$

Using these we get

$$
\begin{aligned}
& X_{\zeta}=V\left(V X_{(0)}^{\bar{\zeta}}+\epsilon \frac{U}{2} h^{\prime \prime \prime}(\zeta) X_{(0)}^{\zeta}\right), \\
& X_{\bar{\zeta}}=V\left(V X_{(0)}^{\zeta}+\epsilon \frac{U}{2} \bar{h}^{\prime \prime \prime}(\bar{\zeta}) X_{(0)}^{\bar{\zeta}}\right) .
\end{aligned}
$$

Here $\epsilon$ is the infinitesimal parameter of conformal transformations. Also note that, unlike the previous case, we have some nonzero surface current leading to

$$
X_{V}=\frac{\epsilon U}{2}\left(\bar{h}^{\prime \prime}(\bar{\zeta}) X_{(0)}^{\bar{\zeta}}+h^{\prime \prime}(\zeta) X_{(0)}^{\zeta}\right) .
$$

Again, it is evident from (3.29) that there is a distortion of the particles after encountering the impulsive gravitational wave and the distortions are determined by the superrotation parameter. Because of the presence of a surface current, this distortion moves the test particles off the spatial two-dimensional (as $X_{V}$ is nonzero) surface where they were initially situated at rest.

\section{MEMORY EFFECT ON NULL GEODESICS}

\section{A. Setup}

Here, we consider a null congruence crossing a null hypersurface $\Sigma$ orthogonally, and study the change in the optical parameters like shear, expansion, etc., We compute these changes for a congruence defined by the tangent vector $N$ as it crosses the IGW. To do so, we first take a null congruence $N_{+}$to the "+" side of the null shell and evaluate the components of it in a common coordinate system installed across the shell. Next, the components of the same vector are evaluated to the "-" side using the matching (soldering) conditions. In this procedure, the geodesic congruence suffers a jump and so as the expansion and shear corresponding to the congruence [29]. The setup is shown in Fig. 1.

For null geodesics, $N^{\mu}$ is already defined in (2.1), which can be thought of as the tangent vector of some hypersurface orthogonal (locally with the null analogue of Gaussian normal coordinates) to null geodesics across $\Sigma$. We denote $N_{-}$to be the tangent to the congruence to the - side of $\Sigma$. Mathematically, the effect of the congruence $N_{+}$crossing the null shell is obtained by applying a coordinate transformation between the coordinates $x_{+}^{\mu}$ to a coordinate $x^{\mu}$ installed locally across the shell [29]. ${ }^{3}$ These relations

\footnotetext{
${ }^{3}$ One could have done the transformation in the "-_ side equivalently.
} 


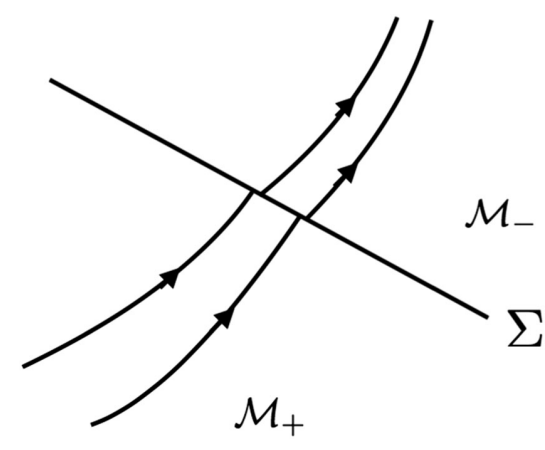

FIG. 1. Null geodesics crossing a null hypersurface $\Sigma$ from $\mathcal{M}_{+}$ to $\mathcal{M}_{-}$. Geodesics experience a jump upon crossing the hypersurface $\Sigma$ represented by soldering transformations.

between coordinates carry the soldering parameters producing memory effect.

Let $N_{0}$ denote the vector $N_{+}$transformed in the common coordinate system and evaluated at the hypersurface $\Sigma$. We take coordinates $x_{-}^{\mu}$ coinciding with $x^{\mu}$. For expressing $N_{0}$ in a common coordinate system one needs the following transformation relation:

$$
N_{0}^{\alpha}(x)=\left.\left(\frac{\partial x_{+}^{\beta}}{\partial x^{\alpha}}\right)^{-1} N_{+}^{\beta}\right|_{\Sigma} .
$$

The inverse Jacobian matrix $\left(\frac{\partial x_{+}^{\beta}}{\partial x^{\alpha}}\right)^{-1}$ is to be evaluated using the ("on-shell") soldering transformations. ${ }^{4}$ The vector in the "-" side is obtained by considering components of $N_{0}$ as initial values. Next, we compute the "failure" tensor $B$ for the vector $N_{0}$ by taking the covariant derivative of it and projecting it on the hypersurface. This tensor encodes the amount of failure for the congruence to remain parallel to each other.

$$
B_{A B}=e_{A}^{\alpha} e_{B}^{\beta} \nabla_{\beta} N_{0 \alpha}
$$

Kinematical decomposition of this tensor leads to two measurable quantities (assuming zero twist for hypersurface orthogonal congruence):

$$
\begin{gathered}
\text { Expansion: } \Theta=\gamma^{A B} B_{A B}, \\
\text { Shear: } \Sigma_{A B}=B_{A B}-\frac{\Theta}{2} \gamma_{A B},
\end{gathered}
$$

where $\gamma_{A B}$ is the induced metric on the null shell. Then, the memory of the IGW or shell is captured through the jump

\footnotetext{
${ }^{4} \mathrm{We}$ call the soldering freedom confined on the null hypersurface on shell; see (A1) of Appendix A. The "off-shell" version can be obtained from the on-shell version by extending the coordinate transformations off the surface in the transverse direction; see (A2) of Appendix A.
}

of these quantities across $\Sigma{ }^{5}$ Note that even if we use the on-shell version of the soldering transformations, the jump in these quantities will be captured for a geodesic crossing the shell originating from the + side of the shell.

$$
\begin{aligned}
{[\Theta] } & =\left.\Theta\right|_{\Sigma_{+}}-\left.\Theta\right|_{\Sigma_{-}}, \\
{\left[\Sigma_{A B}\right] } & =\left.\Sigma_{A B}\right|_{\Sigma_{+}}-\left.\Sigma_{A B}\right|_{\Sigma_{-}}
\end{aligned}
$$

Next, we let the test geodesics travel off the shell, and we get additional contributions in jumps of the expansion and shear. This is achieved by transforming $B$ tensor further to the "-" side of the shell via the following relation that is obvious from the hypersurface orthogonal nature of the congruence:

$$
x_{-}^{\mu} \equiv x^{\mu}=x_{0}^{\mu}+U N_{0}^{\mu}\left(x^{\mu}\right)+\mathcal{O}\left(U^{2}\right) .
$$

Note that all the additional contributions in expansion and shear are proportional to $U$, the parameter of the off-shell extension, and would coincide with the jumps when we restrict the transformations on the shell, i.e., for $U=0$. After determining these off-shell transformations we can evaluate the failure tensor. For determining the $B$ tensor off the shell, we first compute $B_{A B}=e_{A}^{\alpha} e_{B}^{\beta} \nabla_{\beta} N_{0 \alpha}$ and then pull it back to a point infinitesimally away from the shell.

$$
\tilde{B}_{A B}\left(x^{\mu}\right)=\frac{\partial x_{0}^{M}}{\partial x^{A}} \frac{\partial x_{0}^{N}}{\partial x^{B}} B_{M N}\left(x_{0}^{\mu}\right) .
$$

In the far region of the shell, the $B$ tensor is evaluated using $N_{+}=\lambda \partial_{U}$, and clearly it will be different from the expression we get from the above equation leading to the jumps in its different components. Next, we show these jumps for shells in Schwarzschild spacetime.

\section{B. Memory for supertranslation-like transformations}

We consider the case of the Schwarzschild black hole in the Kruskal coordinate (2.12). For plane-fronted waves, a similar kind of memory effect has been reported in [29]. Here we extend that for spherical waves. The details of offshell transformations are given in Appendix A. We first compute the failure tensor off the shell using the inverse Jacobian of transformations given in (B1),

$$
\begin{aligned}
N_{0}= & \frac{e}{8 m^{2}}\left(\partial_{U}+\frac{1}{4 F_{V}^{2} m^{2} e}\left(F_{\theta}^{2}+\frac{F_{\phi}^{2}}{\sin ^{2} \theta}\right) \partial_{V}-\frac{F_{\theta}}{2 F_{V} m^{2} e} \partial_{\theta}\right. \\
& \left.-\frac{F_{\phi}}{2 F_{V} m^{2} e \sin ^{2} \theta} \partial_{\phi}\right)\left.\right|_{\Sigma}
\end{aligned}
$$

\footnotetext{
${ }^{5}$ We discard the possibility of generating a nonvanishing twist to the congruence, initially possessing a zero twist, after IGWs pass through it. This is ensured from the evolution equation [29] of twist.
} 
With this and using (B4) one can evaluate $\tilde{B}_{A B}$ off the shell:

$$
\tilde{B}_{A B}=\frac{1}{\operatorname{det}(J)^{2}}\left(\begin{array}{cc}
\left(1+\frac{2 U}{e} T_{\phi \phi}\right)^{2}+\frac{4 U^{2}}{e^{2}} T_{\theta \phi}^{2} & -\frac{2 U}{e} T_{\theta \phi}\left(2+\frac{2 U}{e} T_{\phi \phi}+\frac{2 U}{e} T_{\theta \theta}\right) \\
-\frac{2 U}{e} T_{\theta \phi}\left(2+\frac{2 U}{e} T_{\phi \phi}+\frac{2 U}{e} T_{\theta \theta}\right) & \left(1+\frac{2 U}{e} T_{\theta \theta}\right)^{2}+\frac{4 U^{2}}{e^{2}} T_{\theta \phi}^{2}
\end{array}\right) B_{M N}
$$

The failure tensor $B_{A B}$ for the congruence with $N_{+}=$ $\lambda \partial_{U}^{6}$ yields

$$
B_{A B}=e_{A}^{\alpha} e_{B}^{\beta} \nabla_{\beta} N_{\alpha}=-\Gamma_{B A}^{V} N_{V} .
$$

We find the $B$ tensor before and after the null congruence crosses the shell to be different which means there is a jump. Clearly for $U=0$, the off-shell failure tensor $\tilde{B}$ reduces to $B$ evaluated at the shell $\Sigma$. Now focusing particularly on supertranslation and using (2.15) and (B2), one gets the following jumps across the shell:

$$
\begin{aligned}
{[\Theta] } & =\frac{1}{\left(4 m^{2}\right)^{2}}\left(T_{\theta \theta}+\csc (\theta)^{2} T_{\phi \phi}+T_{\theta} \cot (\theta)\right), \\
{\left[\Sigma_{\theta \theta}\right] } & =\frac{1}{8 m^{2}}\left(T_{\theta \theta}-T_{\phi \phi} \csc ^{2}(\theta)-T_{\theta} \cot (\theta)\right), \\
{\left[\Sigma_{\phi \phi}\right] } & =\frac{1}{8 m^{2}}\left(T_{\phi \phi}-T_{\theta \theta} \sin ^{2}(\theta)+\frac{T_{\theta} \sin (2 \theta)}{2}\right), \\
{\left[\Sigma_{\theta \phi}\right] } & =\frac{1}{(2 m)^{2}}\left(T_{\theta \phi}-T_{\phi} \cot (\theta)\right) .
\end{aligned}
$$

It is apparent from the above expressions that the jumps in different components of $B$ are related to the supertranslation parameter $T(\theta, \phi)$, and we get a memory corresponding to this. We could have got jumps using the off-shell tensor $\tilde{B}$; in that case, the jumps will differ from this by the terms proportional to $U$. It should be noted, the jump in the shear term is arising due to the presence of IGWs while in the expansion it is due to the presence of shell stress tensor.

\section{Memory for superrotation-type transformations}

First, we focus on the flat space which corresponds to $\eta=0, \Lambda=0$ for the metric mentioned in (2.16). For this case, using (2.17) we have
$N_{0}=\left(\partial_{U}-\frac{\epsilon \bar{h}^{\prime \prime}(\bar{\zeta})}{2 V} \partial_{\zeta}-\frac{\epsilon h^{\prime \prime}(\zeta)}{2 V} \partial_{\zeta}\right)+\left.\mathcal{O}\left(\epsilon^{2}\right)\right|_{\Sigma}$

Repeating the similar computation as in Sec. IV B, and using (B5) we get to leading order in $\epsilon$,

$$
\begin{aligned}
{[\Theta] } & =\mathcal{O}\left(\epsilon^{2}\right), \quad\left[\Sigma_{\zeta \zeta}\right]=\frac{\epsilon V h^{\prime \prime \prime}(\zeta)}{2}+\mathcal{O}\left(\epsilon^{2}\right), \\
{\left[\Sigma_{\bar{\zeta} \bar{\zeta}}\right] } & =\frac{\epsilon V \bar{h}^{\prime \prime \prime}(\bar{\zeta})}{2}+\mathcal{O}\left(\epsilon^{2}\right), \quad\left[\Sigma_{\zeta \bar{\zeta}}\right]=\mathcal{O}\left(\epsilon^{2}\right), \quad\left[\Sigma_{\bar{\zeta} \zeta}\right]=\mathcal{O}\left(\epsilon^{2}\right) .
\end{aligned}
$$

Again it is evident that the jumps in these tensors capture the superrotation-type of transformations. We can perform the similar analysis for constant negative and positive curvature spaces which are also described by the class defined in (2.16).

\section{Memory for null shell near a black hole horizon}

In this case, the null surface is situated at $U=\epsilon$ of the Schwarzschild black hole. Here, we use transformation relations mentioned in (2.19). The inverse Jacobian and other details are displayed in Appendix B. Using (B6), the tangent vector of past congruence can be written as

$$
N_{0}=\frac{e-2 V \epsilon}{8 m^{2}}\left(\partial_{U}-B \partial_{\zeta}-\bar{B} \partial_{\bar{\zeta}}\right)+\left.\mathcal{O}\left(h^{2}, \bar{h}^{2}, \epsilon h, \epsilon \bar{h}\right)\right|_{\Sigma} .
$$

Performing a similar analysis as was done in Sec. IV B we get

$$
\begin{gathered}
{[\Theta]=\frac{4 e^{2-m^{2}}\left((1+\zeta \bar{\zeta})\left(h^{\prime}(\zeta)+\bar{h}^{\prime}(\bar{\zeta})\right)-2 \bar{\zeta} h(\zeta)-2 \zeta \bar{h}(\bar{\zeta})\right)}{1+\zeta \bar{\zeta}}+\mathcal{O}\left(h^{2}, \bar{h}^{2}, \epsilon h, \epsilon \bar{h}\right),} \\
{\left[\Sigma_{\zeta \zeta}\right]=-\frac{e}{4(1+\zeta \bar{\zeta})^{2}} \partial_{\zeta} \bar{B}+\mathcal{O}\left(h^{2}, \bar{h}^{2}, \epsilon h, \epsilon \bar{h}\right), \quad\left[\Sigma_{\bar{\zeta} \bar{\zeta}}\right]=-\frac{e}{4(1+\zeta \bar{\zeta})^{2}} \partial_{\bar{\zeta}} B+\mathcal{O}\left(h^{2}, \bar{h}^{2}, \epsilon h, \epsilon \bar{h}\right),} \\
{\left[\Sigma_{\zeta \bar{\zeta}}\right]=\mathcal{O}\left(h^{2}, \bar{h}^{2}, \epsilon h, \epsilon \bar{h}\right), \quad\left[\Sigma_{\bar{\zeta} \zeta}\right]=\mathcal{O}\left(h^{2}, \bar{h}^{2}, \epsilon h, \epsilon \bar{h}\right) .}
\end{gathered}
$$

$B$ and $\bar{B}$ are defined in (A12).

\footnotetext{
${ }^{6} \lambda$ is arbitrary and only equal to $\frac{e}{8 m^{2}}$ on the shell.
} 


\section{DISCUSSIONS}

In violent astrophysical phenomena, gravitational wave pulses with considerable strength are generated. We have modeled such waves as null shells which represent the history of such impulsive gravitational waves. The motivation of this work is to find the BMS memory effect near the vicinity of a black hole horizon. For this, we have reviewed the theory of null shells endowed with BMS-like symmetries. It is well known that these shells, in general, carry the nonzero stress tensor along with IGWs. We have studied the interaction of such IGWs and stress tensor components on test particles. Let us list the findings of this paper:

(i) First, we have studied the horizon shell situated at the horizon of the Schwarzschild black hole carrying IGWs and evaluated its effect on the deviation vector connecting two nearby timelike geodesics crossing the shell. In this case, where no surface current is present, the effect of IGWs on test particles, initially at rest at the two-dimensional spatial surface, is to displace them relative to each other within the surface. This displacement is characterized by a supertranslation parameter indicating the supertranslation memory effect. This work is an extension of earlier works on similar effects for planar gravitational waves [25-28,34].

(ii) Next, we considered the effect of superrotations on the deviation vector again on timelike congruence. We have exhibited the superrotation memory for Penrose's spherical IGW spacetime. In this case, due to the presence of the nonzero surface current, the test particles initially situated at rest on the twodimensional spatial surface will get displaced off the surface. A similar effect is expected to be exhibited by a null shell situated near the event horizon of a black hole. This superrotation-like memory effect near the horizon has not been considered earlier.

(iii) Furthermore, we turned our attention to null geodesics. We have shown the expansion and shear parameters of null geodesic congruence encounter jumps characterized by a supertranslation parameter upon crossing a null shell situated at the Schwarzschild horizon.

(iv) Finally, we have shown that a null shell placed just outside of a black hole horizon exhibits a superrotation-like memory effect on the null observers in a similar fashion. We also indicated a similar effect for Penrose's spherical IGW spacetime.

The memory effect near the horizon of a black hole may offer many minute details of the symmetries appearing at the horizon region. We hope the more advanced versions of present-day detectors might capture the effects considered here. A study of the near horizon analogue of the (far region) memory effect is under progress and will be reported elsewhere. The relation between BMS-like memories and the Penrose limit for exact impulsive wave spacetimes can offer interesting findings [44]. As there exists some modified version of the BMS algebra on a null surface situated at a finite location of a manifold [21], it will be interesting to investigate the role of those symmetries on the gravitational memory effect. The role of BMS-like symmetries in the study of quantum fluctuations of these spherical IGW backgrounds could be a fascinating area of research $[45,46]$. It will also be interesting to see the implications of our results in the context of holography (AdS/CFT correspondence). Finally, understanding the connection between the quantum vacuum structure of gravity and these memories is going to be an active field of study.

\section{ACKNOWLEDGMENTS}

S. B. is supported by SERB-DST through the Early Career Research award Grant No. ECR/2017/002124. The research of A. B. is supported by the JSPS Grant-in-Aid for JSPS fellows (17F17023).

\section{APPENDIX A: OFF-SHELL EXTENSIONS OF SOLDERING FREEDOM ON KILLING HORIZONS}

Here we provide necessary expressions useful for the computation of a stress tensor (2.7) on null shell. The Schwarzschild Killing horizon case has been presented in [22]; here we briefly review that. Let us consider the case of the Schwarzschild black hole mentioned in (2.12). The null shell is located at $U=0$. On the + side we can perform the following transformations:

$$
V_{+}=F(V, \theta, \phi), \quad \theta_{+}=\theta, \quad \phi=\phi_{+} .
$$

We choose the intrinsic coordinates $x^{\mu}$ to be coordinates on the - side. Introducing the following ansatz [22]:

$V_{+}=F(V, \theta, \phi)+U A(V, \theta, \phi), \quad U_{+}=U C(V, \theta, \phi)$,

$\theta_{+}=\theta+U B^{\theta}(V, \theta, \phi), \quad \phi_{+}=\phi+U B^{\phi}(V, \theta, \phi)$,

and demanding the continuity of the spacetime metric across the junction at leading order in $U$, we get

$$
\begin{aligned}
C & =\frac{1}{\partial_{V} F}, \\
A & =\frac{e}{4} \partial_{V} F\left(\left(\frac{2}{e} \frac{\partial_{\theta} F}{\partial_{V} F}\right)^{2}+\sin (\theta)^{2}\left(\frac{2}{e} \frac{1}{\sin (\theta)^{2}} \frac{\partial_{\tilde{\phi}} F}{\partial_{V} F}\right)^{2}\right), \\
B^{\theta} & =\frac{2}{e} \frac{\partial_{\theta} F}{\partial_{V} F}, \quad B^{\phi}=\frac{2}{e} \frac{1}{\sin (\theta)^{2}} \frac{\partial_{\phi} F}{\partial_{V} F} .
\end{aligned}
$$

We then expand the tangential components of the metrics of both sides $\mathcal{M}_{+}$and $\mathcal{M}_{-}$to linear order in $U$ and read off the components $\gamma_{a b}$ using (2.9), $\gamma_{a b}=N^{\alpha}\left[\partial_{\alpha} g_{a b}\right]=$ $N^{U}\left[\partial_{U} g_{a b}\right]$ with $N^{U}=\frac{e}{8 m^{2}}$. We write down different components of the $\gamma$ tensor: 


$$
\begin{aligned}
& \gamma_{V a}=2 \frac{\partial_{V} \partial_{a} F}{\partial_{V} F}, \quad \gamma_{\theta \theta}=2\left(\frac{\nabla_{\theta}^{(2)} \partial_{\theta} F}{\partial_{V} F}-\frac{1}{2}\left(\frac{F}{\partial_{V} F}-V\right)\right), \\
& \gamma_{\theta \phi}=2\left(\frac{\nabla_{\theta}^{(2)} \partial_{\phi} F}{\partial_{V} F}\right), \\
& \gamma_{\phi \phi}=2\left(\frac{\nabla_{\phi}^{(2)} \partial_{\phi} F}{\partial_{V} F}-\frac{1}{2} \sin (\theta)^{2}\left(\frac{F}{\partial_{V} F}-V\right)\right) .
\end{aligned}
$$

Using (A4) and (2.8), we can identify shell-intrinsic objects as follows:

$$
\begin{aligned}
& p=-\frac{1}{16 \pi} \gamma_{V V}=-\frac{1}{8 \pi} \frac{\partial_{V}^{2} F}{\partial_{V} F}, \quad j^{A}=\frac{1}{32 m^{2} \pi} \sigma^{A B} \frac{\partial_{B} \partial_{V} F}{\partial_{V} F}, \\
& \mu=-\frac{1}{32 m^{2} \pi \partial_{V} F}\left(\nabla^{(2)} F-F+V \partial_{V} F\right) .
\end{aligned}
$$

For supertranslation we need to replace $F(V, \theta, \phi)$ by $V+T(\theta, \phi)$. Then only $\mu$ is nonzero,

$$
\mu=-\frac{1}{32 m^{2} \pi}\left(\nabla^{(2)} T(\theta, \phi)-T(\theta, \phi)\right) .
$$

\section{Constant curvature spacetimes}

Now we consider the class of metrics given by (2.16) and extend the symmetry transformations mentioned in (2.17). We start with the following ansatz:

$$
\begin{aligned}
V_{+} & =V\left(1-\frac{\epsilon \tilde{\Omega}(\zeta, \bar{\zeta})}{2}\right)+U A(V, \zeta, \bar{\zeta}), \\
U_{+} & =U C(V, \zeta, \bar{\zeta}), \\
\zeta_{+} & =\zeta+\epsilon h(\zeta)+U B(V, \zeta, \bar{\zeta}), \\
\bar{\zeta}_{+} & =\bar{\zeta}+\epsilon \bar{h}(\bar{\zeta})+U \bar{B}(V, \zeta, \bar{\zeta}),
\end{aligned}
$$

where $\tilde{\Omega}(\zeta, \bar{\zeta})$ is defined in (2.18) and we work up to linear order in $\epsilon$. Again, demanding the continuity of the full spacetime metric across the junction at leading order in $U$ we get

$$
\begin{aligned}
& A=\frac{\epsilon \eta \tilde{\Omega}(\zeta, \bar{\zeta})}{2}, \quad C=1+\frac{\epsilon \tilde{\Omega}(\zeta, \bar{\zeta})}{2}, \\
& B=\frac{\epsilon\left((1+\eta \zeta \bar{\zeta})\left(\bar{h}^{\prime \prime}(\bar{\zeta})(1+\eta \zeta \bar{\zeta})-2 \eta \zeta \bar{h}^{\prime}(\bar{\zeta})\right)+2 \eta^{2} \zeta^{2} \bar{h}(\bar{\zeta})-2 \eta h(\zeta)\right)}{2 V}, \\
& \bar{B}=\frac{\epsilon\left((1+\eta \zeta \bar{\zeta})\left(h^{\prime \prime}(\zeta)(1+\eta \zeta \bar{\zeta})-2 \eta \bar{\zeta} h^{\prime}(\zeta)\right)+2 \eta^{2} \bar{\zeta}^{2} h(\zeta)-2 \eta \bar{h}(\bar{\zeta})\right)}{2 V},
\end{aligned}
$$

where we have used (2.18). Now we can compute the $\gamma_{a b}$ as before. For simplicity, we write the results of a flat spacetime, i.e., $\eta=0, \Lambda=0$. The $\eta, \Lambda \neq 0$ case can be done analogously. So for the flat space we get

$$
\begin{aligned}
& \gamma_{V \zeta}=h^{\prime \prime}(\zeta), \quad \gamma_{V \bar{\zeta}}=\bar{h}^{\prime \prime}(\bar{\zeta}), \\
& \gamma_{\zeta \zeta}=V h^{\prime \prime \prime}(\zeta), \quad \gamma_{\bar{\zeta} \bar{\zeta}}=V \bar{h}^{\prime \prime \prime}(\bar{\zeta}) .
\end{aligned}
$$

Consequently, we will only have nonvanishing currents.

$$
j^{\zeta}=\frac{\epsilon \bar{h}^{\prime \prime}(\bar{\zeta})}{16 \pi V^{2}}, \quad j^{\bar{\zeta}}=\frac{\epsilon h^{\prime \prime}(\zeta)}{16 \pi V^{2}} .
$$

\section{Null surface near Killing horizon}

Lastly, we present the off-shell extension of the symmetry transformations mentioned in (2.19). Keeping only those terms which are linear in $h(\zeta), \bar{h}(\bar{\zeta})$ and $\epsilon$, we get
$U_{+}=(U-\epsilon) A$,

$V_{+}=-\frac{a \tilde{\Omega}(\zeta, \bar{\zeta})}{b \epsilon}+V(1-\tilde{\Omega}(\zeta, \bar{\zeta}))+(U-\epsilon) C$,

$\zeta_{+}=\zeta+h(\zeta)+(U-\epsilon) B$,

$\bar{\zeta}_{+}=\bar{\zeta}+\bar{h}(\bar{\zeta})+(U-\epsilon) \bar{B}$.

$a=4 m^{2}, b=-\frac{8 m^{2}}{e}$ and $\tilde{\Omega}(\zeta, \bar{\zeta})$ is defined in (2.18). Then demanding the continuity of the metric across the null shell up to $\mathcal{O}(U-\epsilon)$ yields

$$
\begin{aligned}
A= & 1+\frac{\left((\zeta \bar{\zeta}+1)\left(h^{\prime}(\zeta)+\bar{h}^{\prime}(\bar{\zeta})\right)-2 \bar{\zeta} h(\zeta)-2 \zeta \bar{h}(\bar{\zeta})\right)}{1+\zeta \bar{\zeta}}, \\
C= & \mathcal{O}\left(h(\zeta)^{2}, \bar{h}(\bar{\zeta})^{2}\right), \\
B= & -2 e^{1-m^{2}}\left(2 h(\zeta)-(\zeta \bar{\zeta}+1)\left((\zeta \bar{\zeta}+1) \bar{h}^{\prime \prime}(\bar{\zeta})\right.\right. \\
& \left.\left.-2 \zeta \bar{h}^{\prime}(\bar{\zeta})\right)-2 \zeta^{2} \bar{h}(\bar{\zeta})\right) \\
\bar{B}= & -2 e^{1-m^{2}}\left(2 \bar{h}(\bar{\zeta})-(\zeta \bar{\zeta}+1)\left((\zeta \bar{\zeta}+1) h^{\prime \prime}(\zeta)\right.\right. \\
& \left.\left.-2 \bar{\zeta} h^{\prime}(\zeta)\right)-2 \bar{\zeta}^{2} h(\zeta)\right) .
\end{aligned}
$$




\section{APPENDIX B: JACOBIAN OF TRANSFORMATIONS}

Here we present the expression for the Jacobian matrix mentioned in (4.1) which is crucial for the computation of the memory effect through (4.5). We use the expressions (A2) and (A3) to get

$$
\left.\left(\frac{\partial x_{+}^{\beta}}{\partial x^{\alpha}}\right)^{-1}\right|_{U=0}=\frac{1}{2 m^{2} e}\left(\begin{array}{cccc}
2 m^{2} e F_{V} & 0 & 0 & 0 \\
\frac{1}{2 F_{V}}\left(F_{\theta}^{2}+\frac{F_{\phi}^{2}}{\sin ^{2} \theta}\right) & \frac{2 m^{2} e}{F_{V}} & -\frac{2 m^{2} e F_{\theta}}{F_{V}} & -\frac{2 m^{2} e F_{\phi}}{F_{V}} \\
-F_{\theta} & 0 & 2 m^{2} e & 0 \\
-\frac{F_{\phi}}{\sin ^{2} \theta} & 0 & 0 & 2 m^{2} e
\end{array}\right) .
$$

For the case of a supertranslation, it further simplifies.

$$
\left.\left(\frac{\partial x_{+}^{\beta}}{\partial x^{\alpha}}\right)^{-1}\right|_{U=0}=\frac{1}{2 m^{2} e}\left(\begin{array}{cccc}
2 m^{2} e & 0 & 0 & 0 \\
\frac{1}{2}\left(T_{\theta}^{2}+\frac{T_{\phi}^{2}}{\sin ^{2} \theta}\right) & 2 m^{2} e & -2 m^{2} e T_{\theta} & -2 m^{2} e T_{\phi} \\
-T_{\theta} & 0 & 2 m^{2} e & 0 \\
-\frac{T_{\phi}}{\sin ^{2} \theta} & 0 & 0 & 2 m^{2} e
\end{array}\right) .
$$

For off shell, we already know that null congruence to the - side is related to the coordinates on the surface via the following expression:

$$
x^{\alpha}=x_{0}^{\alpha}+U N_{0}^{\alpha} .
$$

The $B$ tensor in this mapping would give us the off-shell extended version. The transformation can be written as

$$
\tilde{B}_{A B}=\frac{\partial x_{0}^{M}}{\partial x^{A}} \frac{\partial x_{0}^{N}}{\partial x^{B}} B_{M N} .
$$

$B_{M N}$ is nothing but the components of the $B$ tensor calculated on the shell. We would be considering here only the BMS case.

$$
\frac{\partial x_{0}^{M}}{\partial x^{A}}=\frac{\delta_{A}^{B}}{\left(\delta_{M}^{B}-U \frac{\partial N^{B}}{\partial x^{N}}\right)}=\frac{I}{J} .
$$

Here $J=\left(\delta_{M}^{B}-U \frac{\partial N^{B}}{\partial x^{N}}\right)$, and the inverse of the $J$ matrix is then given by

$$
\left(\delta_{M}^{B}-U \frac{\partial T^{B}}{\partial x^{N}}\right)^{-1}=\frac{1}{\operatorname{det}(J)}\left(\begin{array}{cc}
1+\frac{2 U}{e} T_{\phi \phi} & -\frac{2 U}{e} T_{\theta \phi} \\
-\frac{2 U}{e} T_{\theta \phi} & 1+\frac{2 U}{e} T_{\theta \theta}
\end{array}\right),
$$

where the determinant of $J$ is given by

$$
\operatorname{det}(J)=1+\frac{2 U}{e}\left(T_{\theta \theta}+F_{\phi \phi}\right)-\frac{4 U^{2}}{e^{2}}\left(T_{\theta \phi}^{2}-T_{\theta \theta} T_{\phi \phi}\right) .
$$

Next, using (A7) and (A8) for flat space we get

$$
\left.\left(\frac{\partial x_{+}^{\beta}}{\partial x^{\alpha}}\right)^{-1}\right|_{U=0}=\left(\begin{array}{cccc}
1-\frac{\epsilon}{2}\left(h^{\prime}(\zeta)+\bar{h}^{\prime}(\bar{\zeta})\right) & 0 & 0 & 0 \\
0 & 1+\frac{\epsilon}{2}\left(h^{\prime}(\zeta)+\bar{h}^{\prime}(\bar{\zeta})\right) & \frac{V \epsilon h^{\prime \prime}(\zeta)}{2} & \frac{V \epsilon \bar{h}^{\prime \prime}(\bar{\zeta})}{2} \\
-\frac{\epsilon \bar{h}^{\prime \prime}(\bar{\zeta})}{2 V} & 0 & 1-\epsilon h^{\prime}(\zeta) & 0 \\
-\frac{\epsilon h^{\prime \prime}(\zeta)}{2 V} & 0 & 0 & 1-\epsilon \bar{h}^{\prime}(\bar{\zeta})
\end{array}\right)+\mathcal{O}\left(\epsilon^{2}\right)
$$

For the null surface near the Killing horizon, using (A11) and (A12), we get

$$
\left.\left(\frac{\partial x_{+}^{\beta}}{\partial x^{\alpha}}\right)^{-1}\right|_{U=\epsilon}=\left(\begin{array}{cccc}
1-\frac{\left((\zeta \bar{\zeta}+1)\left(h^{\prime}(\zeta)+\bar{h}^{\prime}(\bar{\zeta})\right)-2 \bar{\zeta} h(\zeta)-2 \zeta \bar{h}(\bar{\zeta})\right)}{1+\zeta \bar{\zeta}} & 0 & 0 & 0 \\
0 & 1+\tilde{\Omega}(\zeta, \bar{\zeta}) & \frac{(a+b V \epsilon) \partial_{\zeta} \tilde{\Omega}(\zeta, \bar{\zeta})}{b \epsilon} & \frac{(a+b V \epsilon) \partial_{\bar{\zeta}} \tilde{\Omega}(\zeta, \bar{\zeta})}{b \epsilon} \\
-B(\zeta, \bar{\zeta}) & 0 & 1-h^{\prime}(\zeta) & 0 \\
-\bar{B}(\zeta, \bar{\zeta}) & 0 & 0 & 1-\bar{h}^{\prime}(\bar{\zeta})
\end{array}\right) .
$$


[1] Y. B. Zeldovich and A. G. Polnarev, Radiation of gravitational waves by a cluster of superdense stars, Sov. Astron. 18, 17 (1974).

[2] V. B. Braginsky and L. P. Grishchuk, Kinematic resonance and the memory effect in free mass gravitational antennas, Sov. Phys. JETP 62, 427 (1985); V. B. Braginsky and K. S. Thorne, Present status of gravitational-wave experiments, in Proceedings of the Ninth International Conference on General Relativity and Gravitation, Jena, 1980 (Cambridge University Press, Cambridge, England, 1983), pp. 239-253.

[3] D. Christodoulou, Nonlinear Nature of Gravitation and Gravitational-Wave Experiments, Phys. Rev. Lett. 67, 1486 (1991).

[4] K. S. Thorne, Gravitational-wave bursts with memory: The Christodoulou effect, Phys. Rev. D 45, 520 (1992).

[5] J. Frauendiener, Note on the memory effect, Classical Quantum Gravity 9, 1639 (1992).

[6] L. Bieri and D. Garfinkle, Perturbative and gauge invariant treatment of gravitational wave memory, Phys. Rev. D 89, 084039 (2014).

[7] A. Strominger and A. Zhiboedov, Gravitational memory, BMS supertranslations and soft theorems, J. High Energy Phys. 01 (2016) 086.

[8] A. Strominger, Lectures on the infrared structure of gravity and gauge theory, arXiv:1703.05448.

[9] P. D. Lasky, E. Thrane, Y. Levin, J. Blackman, and Y. Chen, Detecting Gravitational-Wave Memory with LIGO: Implications of GW150914, Phys. Rev. Lett. 117, 061102 (2016).

[10] M. Favata, Post-Newtonian corrections to the gravitationalwave memory for quasicircular, inspiralling compact binaries, Phys. Rev. D 80, 024002 (2009); The gravitationalwave memory effect, Classical Quantum Gravity 27, 084036 (2010).

[11] A. Lasenby, Black holes and gravitational waves, Proceedings of the Royal Society Workshop on Black Holes, Chichley Hall, UK, 2017 and KIAA, Beijing, 2017.

[12] A. G. Wiseman and C. M. Will, Christodoulou's nonlinear gravitational wave memory: Evaluation in the quadrupole approximation, Phys. Rev. D 44, R2945 (1991).

[13] D. A. Nichols, Spin memory effect for compact binaries in the post-Newtonian approximation, Phys. Rev. D 95, 084048 (2017).

[14] H. Yang and D. Martynov, Testing Gravitational Memory Generation with Compact Binary Mergers, Phys. Rev. Lett. 121, 071102 (2018).

[15] Bondi, M. van der Burg, and A. Metzner, Gravitational waves in general relativity. 7. Waves from axisymmetric isolated systems, Proc. R. Soc. A 269, 21 (1962); R. Sachs, Gravitational waves in general relativity. 8. Waves in asymptotically flat space-times, Proc. R. Soc. A 270, 103 (1962).

[16] S. W. Hawking, M. J. Perry, and A. Strominger, Soft Hair on Black Holes, Phys. Rev. Lett. 116, 231301 (2016).

[17] S. W. Hawking, M. J. Perry, and A. Strominger, Superrotation charge and supertranslation hair on black holes, J. High Energy Phys. 05 (2017) 161.

[18] G. Barnich and C. Troessaert, Symmetries of Asymptotically Flat Four-Dimensional Spacetimes at Null Infinity Revisited, Phys. Rev. Lett. 105, 111103 (2010).
[19] L. Donnay, G. Giribet, H. A. Gonzalez, and M. Pino, Supertranslations and Superrotations at the Black Hole Horizon, Phys. Rev. Lett. 116, 091101 (2016).

[20] L. Donnay, G. Giribet, H. A. Gonzalez, and M. Pino, Extended symmetries at the Black Hole horizon, J. High Energy Phys. 09 (2016) 100.

[21] V. Chandrasekaran, E. E. Flanagan, and K. Prabhu, Symmetries and charges of general relativity at null boundaries, J. High Energy Phys. 11 (2018) 125.

[22] M. Blau and M. O'Loughlin, Horizon shells and BMS-like soldering transformations, J. High Energy Phys. 03 (2016) 029; Horizon shells: Classical structure at the horizon of a Black Hole, Int. J. Mod. Phys. D 25, 1644010 (2016).

[23] S. Bhattacharjee and A. Bhattacharyya, Soldering freedom and Bondi-Metzner-Sachs-like transformations, Phys. Rev. D 98, 104009 (2018).

[24] L. Donnay, G. Giribet, H. A. Gonzlez, and A. Puhm, Black hole memory effect, Phys. Rev. D 98, 124016 (2018).

[25] P.-M. Zhang, C. Duval, G. W. Gibbons, and P. A. Horvathy, Soft gravitons and the memory effect for plane gravitational waves, Phys. Rev. D 96, 064013 (2017).

[26] P.-M. Zhang, C. Duval, G. W. Gibbons, and P. A. Horvathy, The memory effect for plane gravitational waves, Phys. Lett. B 772, 743 (2017).

[27] P.-M. Zhang, C. Duval, and P. A. Horvathy, Memory effect for impulsive gravitational waves, Classical Quantum Gravity 35, 065011 (2018).

[28] R. Steinbauer, The memory effect in impulsive plane waves: Comments, corrections and clarifications, Classical Quantum Gravity 36, 098001 (2019).

[29] M. O'Loughlin and H. Demirchian, Geodesic congruences, impulsive gravitational waves and gravitational memory, Phys. Rev. D 99, 024031 (2019).

[30] R. Penrose, The geometry of impulsive gravitational waves, in General Relativity, Papers in Honour of J. L. Synge (Clarendon Press, Oxford, 1972), pp. 101-115; Y. Nutku and R. Penrose, On impulsive gravitational waves, Twistor Newsletter 34, 9 (1992), http://people.maths.ox.ac.uk/ lmason/Tn/.

[31] C. Barrabes and W. Israel, Thin shells in general relativity and cosmology: The lightlike limit, Phys. Rev. D 43, 1129 (1991).

[32] E. Poisson, A reformulation of the Barrabes-Israel null shell formalism, arXiv:gr-qc/0207101.

[33] C. Barrabes and P. A. Hogan, Singular Null Hypersurfaces in General Relativity (World Scintific Publishing Co., Inc., River Edge, 2003), Light-like signals from violent astrophysical events.

[34] C. Barrabes and P. A. Hogan, Detection of impulsive lightlike signals in general relativity, Int. J. Mod. Phys. D 10, 711 (2001).

[35] A. Strominger and A. Zhiboedov, Superrotations and black hole pair creation, Classical Quantum Gravity 34, 064002 (2017).

[36] H. Balasin, Geodesics for impulsive gravitational waves and the multiplication of distributions, Classical Quantum Gravity 14, 455 (1997).

[37] R. Steinbauer, Geodesics and geodesic deviation for impulsive gravitational waves, J. Math. Phys. (N.Y.) 39, 2201 (1998); On the geometry of impulsive gravitational waves, arXiv:gr-qc/9809054. 
[38] J. Podolosky, Exact impulsive gravitational waves in spacetimes of constant curvature, in Gravitation: Following the Prague Inspiration (World Scientific Publishing Co., Singapore, 2002), pp. 205-246; J. Podolsky and J. B. Griffiths, Expanding impulsive gravitational waves, Classical Quantum Gravity 16, 2937 (1999); Nonexpanding impulsive gravitational waves with an arbitrary cosmological constant, Phys. Lett. A 261, 1 (1999); M. Ortaggio and J. Podolsky, Impulsive waves in electrovac direct product space-times with Lambda, Classical Quantum Gravity 19, 5221 (2002); J. Podolsky and M. Ortaggio, Symmetries and geodesics in (anti-)de Sitter space-times with nonexpanding impulsive waves, Classical Quantum Gravity 18, 2689 (2001); J. Podolsky and R. Steinbauer, Geodesics in space-times with expanding impulsive gravitational waves, Phys. Rev. D 67, 064013 (2003).

[39] R. Steinbauer, Every Lipschitz metric has $C^{1}$-geodesics, Classical Quantum Gravity 31, 057001 (2014).

[40] J. Podolsky, C. Smann, R. Steinbauer, and R. Svarc, The global existence, uniqueness and $C^{1}$-regularity of geodesics in nonexpanding impulsive gravitational waves, Classical Quantum Gravity 32, 025003 (2015).
[41] J. Podolsky, C. Smann, R. Steinbauer, and R. Svarc, Cut-and-paste for impulsive gravitational waves with $\Lambda$ : The geometric picture, Phys. Rev. D 100, 024040 (2019).

[42] J. Podolsky, C. Smann, R. Steinbauer, and R. Svarc, The global uniqueness and $C^{1}$-regularity of geodesics in expanding impulsive gravitational waves, Classical Quantum Gravity 33, 195010 (2016).

[43] J. Podolsky and R. Svarc, Refraction of geodesics by impulsive spherical gravitational waves in constant-curvature spacetimes with a cosmological constant, Phys. Rev. D 81, 124035 (2010).

[44] G. M. Shore, Memory, Penrose limits and the geometry of gravitational shockwaves and gyratons, J. High Energy Phys. 12 (2018) 133.

[45] M. Hortacsu, Quantum fluctuations in the field of an impulsive spherical gravitational wave, Classical Quantum Gravity 7, L165 (1990); Erratum9, 799 (1992).

[46] M. Hortacsu, Vacuum fluctuations for spherical gravitational impulsive particles, Classical Quantum Gravity 13, 2683 (1996). 